Ingeniare. Revista chilena de ingeniería, vol. 18 No 1, 2010, pp. 15-25

\title{
GRADO DE SUPERVISIÓN COMO VARIABLE MODERADORA ENTRE LIDERAZGO Y SATISFACCIÓN, MOTIVACIÓN Y CLIMA ORGANIZACIONAL
}

\author{
DEGREE OF SUPERVISION AS A MODERATING VARIABLE OF THE \\ RELATION BETWEEN LEADERSHIP AND SATISFACTION, MOTIVATION AND \\ ORGANIZATIONAL CLIMATE
}

\author{
Alejandro Adrián Cuadra-Peralta ${ }^{1} \quad$ Constanza Beatriz Veloso-Besio $^{1}$ \\ Recibido 5 de agosto de 2008, aceptado 23 de noviembre de 2009 \\ Received: August 5, 2008 Accepted: November 23, 2009
}

\begin{abstract}
RESUMEN
El objetivo de esta investigación es mostrar el impacto del Grado de Supervisión como variable moderadora de la relación entre liderazgo, motivación, satisfacción y clima organizacional. En este estudio, Grado de Supervisión se conceptualizó como un constructo con dos polos, estrecho o lejano. De acuerdo con esto, se dará una supervisión estrecha cuando el líder pase mucho tiempo con sus colaboradores y tenga gran influencia sobre cómo ejecuten su trabajo. El caso contrario daría cuenta de una supervisión lejana. Los resultados encontrados evidencian que el impacto del liderazgo sobre variables como la motivación, satisfacción y clima laboral está fuertemente mediada por Grado de Supervisión. Finalmente, se discute la relevancia de este efecto moderador en el ejercicio del liderazgo de los ingenieros que ejercen tanto una supervisión directa como indirecta.
\end{abstract}

Palabras clave: Grado de supervisión, liderazgo, clima laboral, satisfacción laboral.

\section{ABSTRACT}

The objective of this research is to show the impact of the degree of supervision, as moderator variable of the relationship between leadership, motivation, satisfaction and organizational climate. In this study, Degree of Supervision has been conceptualized as a construct with two poles, close or distant. Accordingly, will be closely monitored when the leader passes much time with their employees and have great influence on his work as implemented, the reverse would account for remote monitoring. The results found evidence that the impact of leadership on variables such as motivation, job satisfaction and climate, is strongly mediated by the degree of supervision. Finally, we discuss the relevance of this moderating effect on the exercise of leadership of the engineers who exercise both direct and indirect supervision.

Keywords: Degree of supervision, leadership, organizational climate, job satisfaction.

\section{INTRODUCCIÓN}

En diversas investigaciones realizadas se ha comprobado la relación existente entre liderazgo y variables asociadas a resultados organizacionales. En este sentido se han encontrado correlaciones significativas entre liderazgo y motivación [8, 12, 18, 25, 26, 29], satisfacción laboral [16, 47] y clima laboral [16]. No obstante, es interesante notar que se haya descuidado en la investigación sobre liderazgo qué tan estrecho o lejano es el ejercicio del liderazgo, variable que llamaremos "grado de supervisión", sobre todo si se considera, intuitivamente, que el impacto que tiene el líder sobre sus colaboradores debería variar en función del tiempo y la influencia que tiene el jefe sobre el trabajo. De acuerdo con esto, un supervisor en terreno, que trabaje codo a codo con su cuadrilla, vigilando y corrigiendo casi cada detalle del trabajo de los operarios, debería tener un fuerte impacto, sea positivo o negativo, sobre aspectos relacionados con la conducta inmediata de los trabajadores, la manera en cómo perciben el clima laboral y el nivel de satisfacción laboral en cada uno de ellos. Ahora bien, un líder que trabaje en otro lugar físico,

\footnotetext{
1 Universidad de Tarapacá. Departamento de filosofía y psicología. 18 de Septiembre 2222. Arica, Chile. E-mail: acuadra@uta.cl; cveloso@uta.cl
} 
que no tenga contacto constante con sus colaboradores $\mathrm{y}$, por ende, no supervise la conducta inmediata de sus subordinados, debería tener un impacto menor sobre estas mismas variables de parte de los trabajadores. Es menester aclarar que lo anterior es independiente de si el líder es considerado y preocupado por los demás o poco considerado, irrespetuoso y poco colaborador. En relación con esto, se tendría que si es considerado, preocupado y la supervisión es estrecha, su impacto será positivo y fuerte, pero si es lejana, su impacto también será bueno, pero más débil. Lo mismo, pero en dirección contraria, debería pasar con el líder poco considerado e irrespetuoso. En concordancia con lo anterior, la importancia de este concepto reside en que después de una extensa revisión bibliográfica, que abarcó diversos estudios metaanalíticos [23, 24, 26, 28], revisiones teóricas [21] y búsqueda en las revistas especializadas sobre liderazgo como: Journal of Organizacional Behavior, Journal of Applied Psychology, The Leadership Quartely, The Journal of Psychology, The International Journal of Organizational Analysis, no se encontró referencia a cómo esta variable o un constructo afín influyen como variable moderadora de la relación entre el liderazgo y las variables que son influidas por el mismo. La búsqueda abarcó los administradores de base de datos EBSCO, PROQUEST y las bases de datos Science Direct, Web of Science, SciELO, Psycinfo.

Grado de Supervisión se considera como un continuo con dos polos, supervisión estrecha a supervisión remota. Para los efectos de esta investigación se define como el tiempo en que se está en contacto directo con la jefatura, el nivel de injerencia que tiene el supervisor en las decisiones y la cercanía física entre líder y colaborador. La razón de su incorporación radica en la hipótesis que Grado de Supervisión cumpliría una función moderadora de la relación entre liderazgo y la motivación, la satisfacción y el clima organizacional, vale decir, esta variable afectaría la intensidad de la covariación entre ambas variables.

\section{LIDERAZGO TRANSFORMACIONAL Y TRANSACCIONAL}

Tanto el enfoque transformacional como el transaccional han sido objeto de estudio durante las dos últimas décadas, siendo consideradas una de las tipologías más aceptadas para clasificar el liderazgo [25, 45].

Si bien la introducción y conceptualización de ambos constructos se debe a Burns (1978), es Bass (1985) quien utiliza el trabajo de aquél para construir su teoría de liderazgo transformacional, pero con diversas modificaciones y elaboraciones [20, 25].
Ahora bien, la diferencia entre un estilo transaccional y uno transformacional radica en lo que los líderes y seguidores ofrecen unos a otros. Los líderes transformacionales ofrecen una visión y se enfocan en necesidades internas de orden mayor. En contraste, los líderes transaccionales se focalizan en el adecuado intercambio de recursos [14]. Es necesario agregar que, si bien ambos estilos tienen características distintivas, no representan los extremos de un continuo (en el que si un líder tiene estrategias transformacionales altas, se espera que las otras conductas sean bajas), sino que son dimensiones complementarias, vale decir, los mejores líderes deberían conjugar los dos.

Una forma final es el estilo laissez-faire o no liderazgo [6, 23] -también denominado Política de no Intervención [45]- que resulta en la ausencia o evitación del liderazgo, vale decir, abdican a sus responsabilidades, son reacios a tomar decisiones, vacilan al tomar acciones y están ausentes cuando se les necesita. Representa la carencia de cualquier liderazgo y debe ser tratado como una entidad independiente $[2,7]$.

\section{DIMENSIONES CLÁSICAS DEL LIDERAZGO: CONSIDERACIÓN E INICIACIÓN DE ESTRUCTURA}

Históricamente, las llamadas teorías clásicas del liderazgo, incluyendo en ellas las teoría de las conductas y las de contingencia, se han basado en las dimensiones de orientación a las personas o consideración y la orientación a la tarea o iniciación de estructura, que fueron postuladas casi en forma paralela por los equipos de las Universidades de Michigan y la Estatal de Ohio, por la importancia que tienen dichas dimensiones, se ha decidido incluirlas en el presente estudio.

Iniciación de Estructura, que se relaciona con el grado en que el supervisor (líder) facilita o define las interacciones del grupo encaminadas a la obtención de la meta, vale decir, incluyen estándares definidos de ejecución, confirman que los subordinados comprendan órdenes y tareas, que sigan los procedimientos operativos establecidos, asignan tareas concretas y enfatizan el cumplimiento de plazos; y Consideración, que alude al grado en el cual el líder muestra preocupación y respeto por sus seguidores, busca su bienestar y les expresa apreciación y apoyo. Ahora bien, un estilo de liderazgo que obtiene alta puntuación en Iniciación de Estructura y Consideración dará lugar a resultados positivos [26]. No obstante, es importante indicar que, hasta el advenimiento de la teoría de liderazgo transformacional $[6,11]$, estas dos dimensiones dominaron la investigación, señalándose que "Consideración e 
Iniciación de Estructura han probado estar entre los más robustos conceptos de liderazgo" [17].

Por otra parte, ambas dimensiones han sido objeto de investigación, estableciéndose asociaciones significativas con indicadores asociados a resultados organizacionales [25], tema que será tratado con más detalle en el apartado siguiente.

\section{LIDERAZGO Y SU INFLUENCIA EN EL CLIMA, SATISFACCIÓN Y MOTIVACIÓN LABORAL}

Se ha comprobado que los cimientos de un buen clima laboral (percepciones construidas y compartidas por los miembros de una organización, respecto de sus políticas) se relacionan, en términos generales, con el adecuado desempeño de la organización, y más específicamente con los siguientes indicadores: conciliación del trabajo con la vida familiar, prestaciones de tipo social, satisfacción en el puesto de trabajo y calidad directiva (liderazgo), entre otros. En relación a este último, se señala que goza de tal relevancia que, sin duda, es el aspecto que más incide en el clima [5]. En esta misma perspectiva, se indica que el estilo de liderazgo que exhiba la jefatura influirá sobre el clima y que éste, a su vez, lo hará sobre la creatividad y productividad [37]. Además, los líderes transformacionales comunican una visión a sus subordinados e incrementan el compromiso de éstos con la visión, mediante la calidad de las relaciones interpersonales. La apertura y la cercanía serían un resultado de éstas y deberían tener un efecto sustancial en el clima percibido [48].

En otra investigación se encontraron correlaciones significativas entre clima y liderazgo transformacional $(0,67)$, clima y liderazgo transaccional $(0,57)$, clima y consideración $(0,66)$ y clima e iniciación de estructura $(0,56)$ [16].

Otro estudio [9] examinó las relaciones entre estilos de liderazgo -transformacional y transaccional-y calidad de clima, entre otros, en contextos I+D y administrativos. Los resultados indican que el liderazgo transformacional en contextos de equipos y unidades de investigación y desarrollo (I+D) explica significativamente la calidad del clima $(0,65)$, mientras que el componente transaccional no logró tener significación estadística. En los ambientes administrativos, el estilo transformacional logró dar cuenta de la calidad del clima $(0,47)$, pero en menor grado que el contexto anterior. El componente transaccional también lo hizo, pero en menor medida.
La satisfacción laboral (o satisfacción con el trabajo) es una actitud relacionada con el trabajo, que ha sido objeto de muchas investigaciones [45]. Dicha actitud puede referirse al trabajo en general o a facetas específicas del mismo. Dentro de esta última postura, se establece que este es un constructo multidimensional que comprende aspectos particulares de la satisfacción relacionada con el pago, el trabajo, la supervisión, las oportunidades profesionales, los beneficios, las prácticas organizacionales y las relaciones con los demás trabajadores [34].

En la actualidad también se tiene en cuenta la naturaleza claramente psicosocial de este proceso, es decir, la interacción del individuo con los factores de su ambiente. De esta manera, se manifiesta que frente a los que defienden que la satisfacción laboral es una cuestión que depende de la personalidad de los trabajadores, hay autores que postulan que lo que realmente influye en el grado de satisfacción es la interpretación que hace el sujeto de las circunstancias de su ambiente de trabajo, interpretación en la que tienen injerencia tanto los factores personales como los del entorno, entre ellos la calidad del liderazgo [48].

Dentro de los estudios realizados que analizan la relación entre las variables objeto de este apartado, se tiene una investigación metaanalítica [25] que recopila una vasta cantidad de estudios sobre el tema (87), en la que se examinó la relación de los estilos transformacional, transaccional y laissez-faire con seis criterios de liderazgo. Entre ellos se encontraba satisfacción del seguidor con el trabajo y satisfacción con el líder [9, 35]. De acuerdo con los resultados, los autores encontraron que, por una parte, la correlación entre la estrategia transformacional y satisfacción del colaborador con el trabajo fue de 0,58 $\mathrm{y}$, por otra, la correlación entre el estilo transaccional y satisfacción laboral del colaborador fue de 0,64 , siendo ambas significativas. Otro resultado de interés es la relación entre estos dos estilos de liderazgo y la satisfacción del seguidor respecto del líder. Así se tiene que la fuerza de interacción entre el estilo transformacional y la satisfacción con el líder fue de 0,71 y la correlación entre el estilo transaccional y la variable aludida fue de 0,55 . Por otra parte, la relación de laissez-faire con satisfacción laboral fue de $-0,28$ y de $-0,58$, respecto de satisfacción con el líder. Estos resultados son concordantes con otros estudios, que habían evidenciado que las dimensiones de liderazgo transformacional y transaccional se podían considerar como predictores válidos de la satisfacción laboral del seguidor, así como de su rendimiento en el trabajo [23].

En otro metaanálisis parecido al anterior [26], en el cual se analizó, entre otras cosas, la relación existente entre 
las dimensiones de Consideración y Estructura con satisfacción del seguidor con su trabajo y satisfacción con el líder, se reportó que la correlación entre Consideración y satisfacción del seguidor con su trabajo fue de 0,46 y para satisfacción con el líder fue de 0,78. Para Iniciación de Estructura fue de 0,22 y 0,33 respectivamente, siendo todas significativas.

En otras dos investigaciones [18, 32] que exploraban la injerencia de los comportamientos del jefe sobre tres resultados organizacionales deseables en los empleados, a saber, satisfacción, compromiso organizacional y productividad [31], se encontró que la primera fue la más influenciada por el jefe. Verbigracia, en el primer estudio [18], los análisis de regresión múltiple arrojaron un 29\%, $22 \%$ y $9 \%$, respectivamente.

Estudios más recientes, igualmente, dan cuenta de la relación entre liderazgo y satisfacción. Es así que en una investigación sobre el rol de la supervisión y liderazgo [10] se encuentra una asociación significativa entre liderazgo transformacional y satisfacción laboral general $(0,29)$. Asimismo, en otro trabajo, se encuentran las siguientes correlaciones con satisfacción: liderazgo transformacional 0,53 , transaccional 0,48 , consideración 0,53 e iniciación de estructura 0,46 , siendo todas estadísticamente significativas [16].

En conjunto con la satisfacción laboral, también se ha estudiado el efecto del liderazgo sobre la motivación de los trabajadores, encontrándose una sólida relación en múltiples estudios, con correlaciones que van de 0,32 a 0,59, dependiendo de las diferentes dimensiones o tipo de liderazgo con la cual se estudia.
También hay un sólido cuerpo de investigaciones que se orientan a evaluar el impacto del liderazgo en variables organizacionales más globales, como es la eficacia organizacional [38, 39, 42], los procesos de toma de decisiones organizacionales $[41,43,46]$ y los valores organizacionales [40].

Los principales resultados de la influencia del liderazgo sobre la satisfacción, motivación y clima organizacional se presentan de manera resumida en la Tabla 1.

En síntesis podemos afirmar, basados en los estudios internacionales y locales, que el liderazgo correlaciona positivamente con la satisfacción, motivación y el clima organizacional.

Volviendo al objetivo original de la presente investigación, examinar el efecto moderador de Grado de Supervisión en la relación entre liderazgo y la satisfacción, motivación y clima organizacional, se plantean las siguientes hipótesis:

H1: Grado de Supervisión ejercerá una influencia moderadora en la relación que se da entre liderazgo general y satisfacción laboral.

H2: Grado de Supervisión ejercerá una influencia moderadora en la relación que se da entre liderazgo general y la motivación laboral.

H3: Grado de Supervisión ejercerá una influencia moderadora en la relación que se da entre liderazgo general y el clima organizacional.

Tabla 1. Correlación de liderazgo con satisfacción laboral, clima organizacional y motivación organizacional.

\begin{tabular}{|l|c|c|c|}
\hline Dimensión o tipo de liderazgo & Satisfacción laboral & Motivación laboral & Clima organizacional \\
\hline Consideración & $0,53(\mathrm{a}) ; 46$ (b) & $0,49(\mathrm{a}) ; 50$ (b) & 0,67 (a) \\
\hline Estructura & $0,56(\mathrm{a}) ; 0,22$ (b) & $0,46(\mathrm{a}) ; 0,40(\mathrm{~b})$ & $0,56(\mathrm{a})$ \\
\hline Liderazgo Transformacional & $0,67(\mathrm{a}) ; 0,58(\mathrm{c})$ & $0,48(\mathrm{a}) ; 0,53$ (c) & 0,67 (a) \\
\hline Liderazgo Transaccional & $0,48(\mathrm{a}) ; 0,64(\mathrm{c})$ & $0,32(\mathrm{a}) ; 0,59(\mathrm{c})$ & 0,57 (a) \\
\hline Liderazgo General & $0,70(\mathrm{a})$ & $0,49(\mathrm{a}) ; 0,53$ (c) & 0,70 (a) \\
\hline
\end{tabular}

(a) = Datos obtenidos en muestras locales por Cuadra-Peralta y Veloso-Besio [16].

(b) = Datos obtenidos del metaanálisis de Judge, Piccolo y Ilies [26].

(c) = Datos obtenidos del metaanálisis de Judge y Piccolo [24]. 


\section{MÉTODO}

Universo: Personal de organizaciones pertenecientes al sector público y privado, de las regiones primera (Iquique) y decimoquinta (Arica).

Muestra: Ciento cuarenta y nueve empleados (subalternos) de organizaciones del sector público $(65 \%)$ y privado (35\%) de la ciudad de Arica, tenían un jefe directo. De éstos, el 57\% eran hombres y el 42, 3\% eran mujeres. El promedio de edad de todos los participantes fue de 36 años. La técnica de muestreo empleada fue por conveniencia, buscando ampliar al máximo las organizaciones tanto públicas como privadas existentes en la región. Para lograr este cometido participaron miembros de las Fuerzas Armadas; trabajadores de organizaciones educativas, tales como universidades y colegios; funcionarios públicos y municipales. A su vez, se consideraron distintos ámbitos del sector económico-privado, en especial del área de comercio y servicios.

Variables: Variables Independientes: Liderazgo Transformacional, Liderazgo Transaccional, Consideración e Iniciación de Estructura. Es menester agregar que de la suma simple de estas cuatro variables, se creó otra denominada "Liderazgo General".

Variables Dependientes: Satisfacción Laboral, Motivación Laboral y Clima Organizacional. Para la variable clima, que se compone de 8 dimensiones, se realizó un análisis factorial que evidenció un factor de segundo orden el cual se llamó "Clima General". La segunda variable dependiente se denominó "Satisfacción Laboral General", que se origina de la suma simple de todos los ítems (15) del instrumento que la mide.

Variable Moderadora: Grado de Supervisión. Finalmente, para Grado de Supervisión se calculó el promedio, siendo todos los valores sobre la media, definidos como Supervisión Estrecha y los valores bajo la media, como Supervisión Remota.

Instrumentos: Para medir Grado de Supervisión del Líder se utilizó el instrumento Cercanía de Supervisión (CS) de Cuadra y Veloso, que se anexa. Consta de seis reactivos, utilizando una escala 0-100 en porcentajes, donde el cero por ciento representa "nada" y $100 \%$ equivale a "todo". $\mathrm{Su}$ fiabilidad es de 0,85 y es unifactoria, con un factor que explica el $59,07 \%$ de la varianza.

Para medir Clima Laboral se utilizó un instrumento elaborado por Cuadra y Veloso (2007). Consta de 8 dimensiones, basado en los estudios clásicos de clima organizacional [48]. Cada reactivo se midió a través de una escala Likert de uno ("totalmente en desacuerdo") a cuatro ("totalmente de acuerdo"); Hostilidad, alude al grado de hostilidad percibida de las relaciones entre los miembros de la organización; Bienestar, referido a la percepción que tienen los trabajadores sobre el nivel de bienestar imperante en la organización; Ambiente Laboral, alude al estado de ánimo general imperante en la organización; Conflicto, alude al cómo la organización percibe y enfrenta los conflictos, es decir, si son encarados a tiempo y con la intensidad adecuada; Estructura, es la percepción de organización y planificación del trabajo en la empresa; Reconocimiento, se refiere a la percepción del reconocimiento, al trabajo bien hecho y la relación desempeño-premio; Compañerismo, alude al grado de cooperación y amistad entre los empleados de la organización. Se presentan los coeficientes de fiabilidad, los que van desde 0,79 a 0,92 [16]. Respecto de su validez estructural, se evidenció un factor de segundo orden que explicó el $60 \%$ de la varianza, al que se denominó "Clima General".

Motivación laboral fue evaluada mediante una encuesta tipo Likert construido ex profeso, cuya confiabilidad fue de 0,91 y goza de buena validez factorial, conformando un factor único que explica el $65 \%$ de la varianza.

Para medir Satisfacción se utilizó la Escala General de Satisfacción (Overall Job Satisfaction) y una escala de percepción de niveles de satisfacción en la organización, creada ex profeso, con una confiabilidad de 0,90 y un factor de primer orden que explica el $72 \%$ de la varianza. La Escala General de Satisfacción fue desarrollada por Warr, Cook y Wall (1979), consta de 15 ítems que deben ser valorados en una escala Likert que va desde uno ("muy insatisfecho") a cinco ("muy satisfecho"). Sus propiedades psicométricas fueron evaluadas en una investigación previa [16]. La fiabilidad, estimada mediante el indicador Alfa de Cronbach, va desde 0,85 a 0,88 . Cabe hacer constatar que esta variable enfatiza la actitud personal, a diferencia de la escala de percepción de niveles de satisfacción, que pone el acento en la percepción que tienen los trabajadores sobre el entorno sociolaboral. La correlación entre ambas escalas es de 0,50. El índice de satisfacción global es el promedio de las dos mediciones.

Para medir Liderazgo Transformacional, Transaccional y laissez-faire (no liderazgo) se utilizó una adaptación del Cuestionario Multifactor de Liderazgo (MLQ) forma 5X [16], siendo éste uno de los instrumentos más utilizados para evaluar liderazgo transformacional [15, 20]. Los indicadores de fiabilidad obtenidos en la adaptación 
realizada anteriormente fueron de 0,87 para liderazgo transformacional y de 0,83 para liderazgo transaccional. Cada ítem se presenta en una escala Likert de uno ("nunca") a cinco ("con mucha frecuencia o siempre").

Para medir Consideración e Iniciación de Estructura se utilizó el Cuestionario de Descripción de la Conducta del Líder (LBDQ) de Halpin (1957), el cual se tradujo y adaptó en una investigación previa [16]. El análisis de consistencia interna arrojó un Alfa de 0,94 para Consideración y de 0,86 para Estructura. En este instrumento cada ítem debía ser valorado mediante la elección de una opción que va de uno ("nunca") a cinco ("con mucha frecuencia o siempre"). Los criterios de elección que lo convirtieron en la mejor opción a la hora de evaluar estos conceptos fueron: su facilidad de aplicación (contiene menos reactivos que la forma XII) y características psicométricas acordes a los estándares exigidos [26].

Diseño y Procedimiento: Se utilizó un diseño ex-post facto, con variables intervalares. Para la recolección de los datos, se le proporcionó a cada participante un cuadernillo que contenía todos los instrumentos mencionados para que los respondiesen de forma anónima. Los datos se analizaron mediante correlación de Pearson y la prueba de diferencia entre dos coeficientes de correlaciones independientes de Preacher [49].

\section{RESULTADOS}

En la Tabla 2 se muestran los resultados de las correlaciones entre las dimensiones generales de liderazgo. Se puede observar que los cuatro estilos correlacionan significativamente entre sí, siendo la correlación más alta entre liderazgo transformacional y consideración $(0,85)$ y la más baja entre liderazgo transaccional y estructura $(0,41)$. No obstante, es importante recordar que un índice de correlación de 0,41 es considerado como moderado. Asimismo, estas correlaciones se ven reflejadas cuando se hace un análisis factorial con estos cuatro estilos. Los resultados evidencian un único factor que explica alrededor del 79\% de la varianza, por lo que es factible considerar "liderazgo general" como una variable en sí misma.

En la Tabla 3 se encuentran las correlaciones de liderazgo general con satisfacción, motivación y clima organizacional, moderado por grado de supervisión, el cual se divide en Supervisión Remota (referido a aquel líder que tiene poco contacto directo con sus empleados y que estaría dado por una supervisión lejana, un bajo nivel de injerencia y poca cercanía física) y Nivel de Supervisión Cercano (referido a aquel líder que frecuentemente tiene contacto directo con sus empleados y que estaría dado por una supervisión cercana, un alto nivel de injerencia y cercanía física). Para obtener la categorización de grado de supervisión se dividió la muestra tomando como base la media obtenida en el instrumento para evaluar grado de supervisión. Para analizar la significación entre las correlaciones se utilizó la prueba de Preacher [49].

Tal como se puede observar en la Tabla 3, las correlaciones entre liderazgo y las variables dependientes estudiadas, se ven claramente afectadas por grado de supervisión. Para explicar mejor este concepto, tomemos como ejemplo la relación entre liderazgo y motivación. Como se puede apreciar, dicha correlación es de 0,49 , lo que explica el $24 \%$ de la varianza. Esto es muy próximo a lo expuesto en la presentación teórica. Pero cuando se incluye la variable moderadora Grado de Supervisión, esta correlación se descompone drásticamente: Cuando la supervisión es remota, dicha correlación es de 0,33 , lo que implica un $11 \%$ de varianza explicada y cuando es estrecha, estos valores suben a 0,66 y $44 \%$, respectivamente. Es decir, la varianza explicada es tres veces mayor, lo que prueba el efecto moderador de Grado de Supervisión. Cabe hacer notar que las variables que se ven más afectadas son motivación, clima de satisfacción, ambiente laboral y compañerismo, no siendo irrelevante el efecto en las otras variables consideradas.

Tabla 2. Correlaciones entre las dimensiones de Liderazgo.

\begin{tabular}{|l|c|c|c|}
\hline & Transformacional & Transaccional & Consideración \\
\hline Transformacional & - & & \\
Transaccional & $0,69^{* *}$ & - & - \\
Consideración & $0,85^{* *}$ & $0,81^{* *}$ & $0,73^{* *}$ \\
Estructura & $0,79^{* *}$ & $0,41^{* *}$ & \\
\hline
\end{tabular}

** Correlación significativa al 0,01. 
Tabla 3. Correlaciones de Liderazgo General Clima, Satisfacción y Motivación, Moderado por Grado de Supervisión.

\begin{tabular}{|c|c|c|c|}
\hline & Clima General & Satisfacción Global & Motivación \\
\hline Liderazgo General & $\begin{array}{c}0,70 \\
(49 \%)\end{array}$ & $\begin{array}{c}0,68 \\
(46 \%)\end{array}$ & $\begin{array}{c}0,49 \\
(24 \%)\end{array}$ \\
\hline Supervisión Remota & $\begin{array}{c}0,62 \\
(39 \%)\end{array}$ & $\begin{array}{c}0,57 \\
(32 \%)\end{array}$ & $\begin{array}{c}0,33 \\
(11 \%)\end{array}$ \\
\hline Supervisión Estrecha & $\begin{array}{c}0,77 \\
(59 \%)\end{array}$ & $\begin{array}{l}0,77 \\
(59 \%)\end{array}$ & $\begin{array}{c}0,66 \\
(44 \%)\end{array}$ \\
\hline $\begin{array}{l}\text { Significación de la diferencia entre las correlaciones } \\
\text { para supervisión remota y estrecha }\end{array}$ & 0,03 & 0,01 & 0,00 \\
\hline
\end{tabular}

Todas las correlaciones evidenciaron significancia estadística al 0,01.

Nota: Los números entre paréntesis corresponden a la varianza explicada.

\section{DISCUSIÓN Y CONCLUSIONES}

El objetivo principal de este estudio era analizar el efecto moderador de grado de supervisión en la relación entre liderazgo y satisfacción, motivación y clima organizacional. Los resultados obtenidos en los análisis confirman el rol modulador de esta nueva variable.

El razonamiento para evaluar la influencia moderadora de grado de supervisión fue considerar que si modificaba la fuerza de la relación existente entre el liderazgo y las variables dependientes, habría un efecto de modulación; en caso contrario, se rechazarían las respectivas hipótesis. Cabe constatar que en los valores de las correlaciones entre liderazgo con satisfacción y motivación laboral, obtenidos en el presente estudio son similares a los obtenidos en los estudios internacionales [8, 12, 16,18, 25, 26, 29, 47]. En cuanto a la relación de liderazgo y clima organizacional, no se encontraron mediciones específicas, fuera de las realizadas por los mismos autores en un estudio anterior [16].

En términos generales se puede afirmar que el objetivo principal fue logrado plenamente, al confirmar cada una de las hipótesis planteadas, las cuales se revisan en detalle a continuación.

En relación a la primera hipótesis Grado de Supervisión ejercerá una influencia moderadora en la relación que se da entre liderazgo general y satisfacción laboral, podemos mantenerla (rechazar la hipótesis nula), por cuanto la correlación general entre liderazgo y satisfacción laboral de 0,68 , que explica $46 \%$ de la varianza y que es similar a la encontrada en estudios internacionales revisados, se descompone drásticamente cuando se hace el análisis según la supervisión sea estrecha o lejana. Cuando la supervisión es estrecha, la correlación entre liderazgo y la satisfacción laboral sube a 0,77 , que equivale al $59 \%$ de la varianza, mientras que cuando el grado de supervisión es lejano, la correlación baja a 0,57 que equivale al $32 \%$ de la varianza, diferencia que es estadísticamente significativa.

En relación a la segunda hipótesis Grado de Supervisión ejercerá una influencia moderadora en la relación que se da entre liderazgo general y la motivación laboral, de acuerdo a los resultados obtenidos en la presente investigación, también se mantiene (se rechaza la hipótesis nula), por cuanto encontramos que la influencia del liderazgo en la motivación que explica el $24 \%$ de la varianza, con una correlación de 0,49 , se ve afectada cuando se introduce la variable grado de supervisión. Cuando la supervisión es estrecha, se explica el $44 \%$ de la varianza, con una correlación de 0,66 , y cuando el grado de supervisión es lejano, liderazgo sólo explica el $11 \%$ de la varianza de la motivación laboral, con una correlación de 0,33 .

Por último, en relación a la tercera hipótesis Grado de Supervisión ejercerá una influencia moderadora en la relación que se da entre liderazgo general y clima organizacional, también se mantiene. Los datos obtenidos en los análisis realizados desglosan la correlación general entre liderazgo y clima de 0,70 , explicando el $49 \%$ de la varianza, en 0,77 cuando la supervisión es estrecha, lo que implica explicar el 59\% de la varianza, y en 0,62 cuando el grado de supervisión es lejano, lo que explica el 39\% de la varianza. Esta diferencia entre las correlaciones es significativa, de acuerdo a la prueba de diferencia entre dos coeficientes de correlaciones independientes de Preacher [49]. 
Por tanto, se puede afirmar, provisionalmente, que el grado de supervisión es una importante variable moderadora. De todas maneras, es menester detallar algo más el asunto. Se esperará que, cuando el líder sea considerado, preocupado por los demás y utilice una supervisión estrecha, variables como motivación y satisfacción (o cualquier otra) aumenten significativamente; cuando el líder sea considerado y remoto, se espera una pequeña disminución en los niveles de motivación y satisfacción; cuando el líder sea poco considerado, irrespetuoso, poco colaborador y estrecho, se espera que los niveles de motivación y satisfacción disminuyan ostensiblemente y cuando el líder sea desconsiderado y remoto, se espera una pequeña disminución de las variables aludidas. Como se puede ver, éstas son algunas de las hipótesis que se sugieren analizar en estudios posteriores.

Ahora bien, la gran importancia de este nuevo hallazgo radica en que, de ser replicado y encontrado los mismos resultados por distintos investigadores, se deberían reanalizar todos los estudios que relacionan liderazgo y variables organizacionales a la luz de este nuevo concepto.

Por otra parte, se optó por considerar el liderazgo un fenómeno global, no desglosándolo entre liderazgo transformacional y transaccional, basándose en los planteamientos de importantes investigadores [3-4] que expresan que los mejores líderes son los que exhiben estrategias transformacionales y transaccionales. A su vez, la Teoría de Liderazgo Completo (Full Range Theory), que es claramente una teoría multivariada, explícitamente aboga por la unión de estas estrategias e incluso la dimensión laissez-faire [2]. Algo parecido, ocurriría con las dimensiones de consideración e iniciación de estructura.

Para fundamentar más lo expuesto en el párrafo anterior, en la presente investigación se encontraron altas correlaciones entre los distintos tipos de liderazgo (Tabla 1), siendo muy interesante reflexionar sobre estos resultados. Si las correlaciones son tan altas, evidentemente, es posible hablar del liderazgo como un solo fenómeno, pero no sólo eso, sino que se puede hipotetizar que no es posible ser apreciado como líder en una de las dimensiones si no se tienen las otras cualidades. Es decir, un líder altamente orientado a la tarea sólo será percibido como tal si además es orientado a las personas. Una eventual explicación de ello, sería que no es posible lograr las metas y resultados si no se tiene una buena imagen como líder orientado a las personas y viceversa. Lo mismo podría decirse en relación a las otras dimensiones del liderazgo. Si consideramos que una de las cosas que caracteriza el liderazgo transformacional es la generación de visiones que involucren a los seguidores, orientación a las personas (consideración), orientación a la tarea y liderazgo transaccional (en especial el refuerzo contingente). Esto permite cuestionar la creencia que las dimensiones del liderazgo se puedan compensar entre sí y a hipotetizar que en condiciones normales, cuando una no se da, las otras tampoco. Esto último tiene serias implicaciones para el desempeño de los ingenieros en cuanto a su rol de supervisor, y en la formación de los futuros profesionales, lo que unido a los resultados obtenidos en la dimensión de cercanía al liderazgo (Tabla 2), nos hace poner el acento en las habilidades de liderazgo de los ingenieros jóvenes, ya que muchos de ellos ocuparán cargos de supervisión de primera línea en sus primeros trabajos, y la calidad de su desempeño dependerá en gran medida de su capacidad de influir a sus colaboradores. Es importante destacar que no todas las universidades ponen énfasis en la formación de las competencias asociadas al liderazgo en la formación de los futuros ingenieros, aspecto que debería revisarse a la luz de los trabajos que, como éste, remarcan la influencia del liderazgo sobre la conducta de los subordinados.

El aporte de este estudio, en términos generales, es la incorporación del grado de supervisión como variable moderadora de la relación entre liderazgo y clima, motivación y satisfacción laboral, de la cual no se han reportado evidencias teórico-empíricas en estudios anteriores, siendo la presente investigación pionera en este aspecto.

En relación a las limitaciones de esta investigación, se puede decir que si bien se incluyeron empresas de la primera región (Iquique), el parque empresarial en la decimoquinta (Arica) aún no logra altos niveles de desarrollo, por lo que no se cuentan con muchas organizaciones desde donde obtener una muestra mayor.

Finalmente, se sugiere realizar más investigación sobre grado de supervisión para examinar su rol modulador, así como las relaciones entre supervisión estrecha y remota cuando el líder sea bueno o malo. Asimismo, se aconseja realizar más estudios en Chile que analicen la relación entre liderazgo, clima y satisfacción. Si bien es cierto, los resultados de este estudio van en la misma dirección que los encontrados en otros países, una sola investigación local no es suficiente para determinar si el liderazgo es un fenómeno que sigue un patrón similar a los reportados previamente. 


\section{REFERENCIAS}

[1] L. Ahumada. "Liderazgo y equipos de trabajo, una nueva forma de entender la dinámica organizacional". Ciencias Sociales Online. Vol. I No 1, pp. 53-63. Septiembre 2004. Fecha de consulta: 1 de abril de 2006. URL: www.uvm.cl

[2] B. Avolio. "Full leadership development: Building the Vital Forces in Organizations". Thousand Oaks, CA: Sage. Estados Unidos. 1999.

[3] B.J. Avolio and B.M. Bass. "Individual consideration viewed at multiple levels of análisis: A multi-level framework for examining the diffusion of transformational leadership". Leadership Quartely. Vol. 6 N $^{\circ}$ 2, pp. 199-218. 1995.

[4] B. Avolio, J. Bruce and B. Bass. "Re-examining the components of transformational and transactional leadership using the Multifactor Leadership Questionnaire". Journal of Occupational and Organizational Psychology. Vol. $72 \mathrm{~N}^{\circ} 4$, pp. 441462. Diciembre 1999.

[5] A. Baguer. "Los cimientos de un buen Clima Laboral". 2006. Fecha de consulta: 31 de marzo de 2006. URL: www.chilecapacita.cl

[6] B. Bass. "Leadership and performance beyond expectations". Free Press. New York. Estados Unidos. 1985.

[7] B.M. Bass. "Transformational Leadership: Industry, military, and educational impact". Lawrence Erlbaum Associates. Mahwh. Estados Unidos, pp. 208. 1998.

[8] B.M. Bass, B.J. Avolio, D.I. Jung and Y. Berson. "Predicting Unit Performance by Assessing Transformational and Transactional Leadership". Journal of Applied Psychology. Vol. $88 \mathrm{~N}^{\circ} 2$, pp. 207-218. Abril 2003.

[9] Y. Berson and D. Linton. "An examination of the relationships between leadership style, quality, and employee satisfaction in $\mathrm{R}+\mathrm{D}$ versus administrative environments". R+D Management. Vol. $35 \mathrm{~N}^{\circ} 1$, pp. 51-59. Enero 2005.

[10] J.E. Bono, H. Jackson, G. Vinson and J.P. Muros. "Workplace Emotions: The Role of Supervision and Leadership". Journal of Applied Psychology. Vol. $92 \mathrm{~N}^{\circ}$ 5, pp. 1.357-1.367. September 2007.

[11] J. Burns. "Leadership". Harper \& Row. New York. Estados Unidos. 1978.

[12] L. Chen. "Examining the Effect of Organization Culture and Leadership Behaviors on Organizational
Commitment, Job Satisfaction, and Job Performance at Small Middle-size Firms of Taiwan". The Journal of American Academy Business, Cambridge. September 2004.

[13] J. Conger and B. Benjamin. "Building Leaders: How Successful Companies Develop the Next Generation". Jossey-Bass. San Francisco, CA. Estados Unidos. 1999.

[14] J.A. Conger and R.N. Kanungo. "Charismatic leadership in organizations". Thousand Oaks, CA: Sage, p. 288. 1998.

[15] I. Cuadrado, F. Molero y M. Navas. "El Liderazgo de Hombres y Mujeres: Diferencias en Estilos de Liderazgo y predoctores de Variables de Resultado Organizacional”. Acción Psicológica. Vol. $2 \mathrm{~N}^{\circ} 2$, pp. 115-129. Junio 2003.

[16] A. Cuadra y C. Veloso. "Liderazgo, Clima y Satisfacción Laboral". Revista Universum. Vol. 2 $\mathrm{N}^{\mathrm{o}}$ 22, pp. 40-56. Julio 2007.

[17] A. Fleishman. "Consideration and structure: Another look at their role in leadership research". In "Leadership: The multiple-level approaches". F. Dansereau \& F. J. Yammarino (Eds.). JAI Press, pp. 51-61. Standford. Estados Unidos. 1995.

[18] J. Foong. "Leadership behaviours: effecs on job satisfaction, productivity and organizacional commitment". Journal of Nursing Managment. Vol. 9 No 4, pp. 191-204. Julio 2001.

[19] T.L. Friedman. "The Lexus and the Olive Tree". Anchor Books, p. 469. New York, Estados Unidos. 2000.

[20] K. Heinitz. "Assessing the validity of the Multifactor Leadership Questionnaire. Discussing New Approaches to Leadership". Tesis para optar al grado de doctor. Berlin University. Berlín, Alemania. 2006.

[21] R. Hogan and R.B. Kaiser. "What We Know About Leadership". Review of General Psychology. Vol. $9 \mathrm{~N}^{\circ}$ 2, pp. 169-180. Junio 2005.

[22] C. Hulin and T. Judge. "Jobs Attitudes. Handbook of Psychology". Industrial and Organizational Psychology. Vol. 12, pp. 255-276. 2003.

[23] T.A. Judge and J.E. Bono. "Personality and Transformational and Transactional Leadership: A Meta-Analysis". Journal of Applied Psychology. Vol. 89 No 5, pp. 901-910. October 2004.

[24] T. Judge, J. Bono, R. Ilies and M. Gerhardt. "Personality and Leadership: A Qualitative and Quantitative Review". Journal of Applied Psychology. Vol. $87 \mathrm{~N}^{\circ}$ 4, pp. 765-780. August 2002. 
[25] T. Judge and R. Piccolo. "Transformational and Transactional Leadership: A Meta-Analytic Test of Their Relative Validity". Journal of Applied Psychology. Vol. 89 No 5, pp. 755-768. February 2004.

[26] T. Judge, R. Piccolo and R. Ilies. "The forgotten ones The Validity of Consideration and Iniciating Structure in Leadership Research". Journal of Applied Psychology. Vol. $89 \mathrm{~N}^{\mathrm{o}}$ 1, pp. 36-51. Febrero 2004.

[27] J.M. Kouzes and B.Z. Posner. "The Leadership Challenge”. Jossey-Bass. San Francisco. Estados Unidos, p. 496. December 1998.

[28] K. Lowe, K. Krocck and N. Sivasubramaniam. "Effectiveness correlates of transformational and transactional leadership: A meta-analytic review of the MLQ literature". Leadership Quartely. Vol. 7 No 3, pp. 385-425. 1996.

[29] R.J. Masi and R.A. Cooke. "Effects of Transformational Leadership on Subordinate Motivation, Empowering and Organizational Productivity". International Journal of Organizational Analysis. Vol. $8 \mathrm{~N}^{\mathrm{o}}$ 1, pp. 16-47. 2000.

[30] W. McCall. "High Flyers: Developing the Next Generation of Leaders". Harvard Business School Press. Boston, MA. Estados Unidos. 1998.

[31] D. McNeese-Smith. "Job satisfaction, productivity and organizational commitment, the result of leadership". Journal of Nursing Administration. Vol. 25, pp. 17-26. 1995.

[32] D. McNeese-Smith. "The influence of manager behavior on nurses' job satisfaction, productivity and comminment". Journal of Nursing Administration. Vol. 27 No 9. pp. 47-55. September 1997.

[33] Ministerio del Trabajo y Asuntos Sociales de España. 2006. Fecha de consulta: 31 de marzo de 2006. URL: www.mtas.es

[34] T. Misener, K. Haddock, J.U. Gleaton and A.R.A. Ajamieh. "Toward and international measure of job satisfaction". Nursing Research. Vol. 45, pp. 87-91. 1996.

[35] M. Olsson and J. Wass. "Leadership styles and actions routines: best practice manufacturing and $\mathrm{R}+\mathrm{D}$ projects". International Journal of Technology Management. Vol. 22 No 4, pp. 327-343. 2001.

[36] K. Parry and P. Sinha. "Resarshing the Trainability of Transformational Organizational Leadership". Human Resource Development International. Vol. $8 \mathrm{~N}^{\mathrm{o}}$ 2, pp. 165-183. Junio 2005.
[37] L. Pedraja y E. Rodríguez. "Efectos del Estilo de Liderazgo sobre la Eficacia de las Organizaciones Públicas". Revista de la Facultad de IngenieríaUniversidad de Tarapacá. Vol. 12 № 2, pp. 63-73. Diciembre 2004.

[38] L. Pedraja-Rejas y E. Rodríguez-Ponce. "Estudio comparativo de la Influencia del estilo de liderazgo y la congruencia de valores en la eficacia de empresas privadas e Instituciones Públicas". INCI. Vol. 33 N 1, pp. 08-13. Enero 2008.

[39] L. Pedraja-Rejas, E. Rodríguez-Ponce, M. BarredaOlavarría, O. Sagredo-Núñez y C. Segovia-León. "Estilos de liderazgo y resultado del sistema de medición de la calidad de la educación: un estudio empírico en los colegios básicos de la ciudad de Arica-Chile". Ingeniare. Revista chilena de ingeniería. Vol. $17 \mathrm{~N}^{\circ}$ 1, pp. 21-26. Abril 2009. URL: http://www.scielo.cl/scielo. php? script=sci_pdf \&pid=S0718-330520090001 00003\&lng=es\&nrm=iso\&tlng=es. ISSN 0718-3305. Doi: 10.4067/S0718-33052009000=es100003.

[40] L. Pedraja-Rejas, E. Rodríguez-Ponce, M. Delgado-Almonte and J. Rodríguez-Ponce. "Transformational and Transactional Leadership: a study of their influence in small companies". Ingeniare. Revista Chilena de Ingeniería. Vol. 14 No 2, pp. 159-166. Agosto 2006.

[41] L. Pedraja-Rejas, E. Rodríguez-Ponce y J. Rodriguez-Ponce. "Liderazgo y decisiones estratégicas: Una perspectiva integradora”. INCI. Vol. $31 \mathrm{~N}^{\circ}$ 8, pp. 577-582. Agosto 2006.

[42] L. Pedraja-Rejas, E. Rodríguez-Ponce y J. Rodríguez-Ponce. "Importancia de los estilos de liderazgo sobre la eficacia: un estudio comparativo entre grandes y pequeñas y medianas empresas privadas". Revista de Ciencias Sociales. Vol. 14 $\mathrm{N}^{\mathrm{o}}$ 1, pp. 20-29. Abril 2008.

[43] L. Pedraja-Rejas, E. Rodríguez-Ponce y J. Rodríguez-Ponce. "Valores Directivos: Sus Efectos Sobre El Diseño e Implementación De La Estrategia”. Ingeniare. Revista Chilena de Ingeniería. Vol. $16 \mathrm{~N}^{\circ}$ 2, pp. 295-300. Septiembre 2008.

[44] J. Peiró. "Psicología de la Organización". Universidad Nacional de Educación a Distancia, pp. 159,165-170. Madrid, España. 2000.

[45] S. Robbins. "Comportamiento Organizacional”. Pearson Education, 10ma Edición. 2004.

[46] E. Rodríguez-Ponce. "Estilos de liderazgo, toma de decisiones estratégicas y eficacia: un estudio empírico en pequeñas y medianas empresas". 
Interciencia. Vol. $32 \mathrm{~N}^{\circ}$ 8, pp. 522-528. Agosto 2007.

[47] G. Topa, M. Depolo y J.F. Morales. "Acoso Laboral: Metaanálisis y modelo integrador de sus antecedentes y consecuencias". Psicothema. Vol. $19 \mathrm{~N}^{\circ}$ 1, pp. 88-84. 2007.

[48] D. Zohar and G. Luria. "Climate as a SocialCognitive Construction of Supervisory Safety
Practices: Scripts as Proxy of Behaviour Patterns". Journal Applied of Psychology. Vol. $89 \mathrm{~N}^{\mathrm{o}} 2$, pp. 322-333. February 2004.

[49] K. J. Preacher. Calculation for the test of the difference between two independent correlation coefficients [Computer software]. URL: http:// www.quantpsy.org. 1 de mayo de 2002.

Anexo 1: Test de evaluación del grado de supervisión

\section{INSTRUCCIONES}

Lea cuidadosamente cada pregunta/afirmación y responda a conciencia cada una de ellas.

\begin{tabular}{|c|c|c|c|c|c|c|c|c|c|c|}
\hline \multicolumn{11}{|c|}{ 1. ¿Qué porcentaje de la jornada de trabajo usted está en contacto directo con su jefe? } \\
\hline Nada & & & & & & & & & & Todo el día \\
\hline $0 \%$ & $10 \%$ & $20 \%$ & $30 \%$ & $40 \%$ & $50 \%$ & $60 \%$ & $70 \%$ & $80 \%$ & $90 \%$ & $100 \%$ \\
\hline \multicolumn{11}{|c|}{ 2. ¿En qué grado su jefe se inmiscuye en las actividades laborales que usted realiza? } \\
\hline Ninguna & & & & & & & & & & Absoluta \\
\hline $0 \%$ & $10 \%$ & $20 \%$ & $30 \%$ & $40 \%$ & $50 \%$ & $60 \%$ & $70 \%$ & $80 \%$ & $90 \%$ & $100 \%$ \\
\hline \multicolumn{11}{|c|}{$\begin{array}{l}\text { 3. Evalúe qué tanta supervisión tiene normalmente la gente que ocupa cargos similares al suyo (independiente de cómo lo } \\
\text { haga su jefe) }\end{array}$} \\
\hline Nada & & & & & & & & & & Muy estrecha \\
\hline $0 \%$ & $10 \%$ & $20 \%$ & $30 \%$ & $40 \%$ & $50 \%$ & $60 \%$ & $70 \%$ & $80 \%$ & $90 \%$ & $100 \%$ \\
\hline \multicolumn{11}{|c|}{ 4. ¿Qué porcentaje de su trabajo es supervisado directamente por su jefe? } \\
\hline Ninguno & & & & & & & & & & Todos \\
\hline $0 \%$ & $10 \%$ & $20 \%$ & $30 \%$ & $40 \%$ & $50 \%$ & $60 \%$ & $70 \%$ & $80 \%$ & $90 \%$ & $100 \%$ \\
\hline \multicolumn{11}{|c|}{ 5. ¿Qué porcentaje de las decisiones que usted toma deben ser consultadas con su jefatura? } \\
\hline Ninguna & & & & & & & & & & Todas \\
\hline $0 \%$ & $10 \%$ & $20 \%$ & $30 \%$ & $40 \%$ & $50 \%$ & $60 \%$ & $70 \%$ & $80 \%$ & $90 \%$ & $100 \%$ \\
\hline \multicolumn{11}{|c|}{ 6. ¿Qué tan cerca físicamente trabaja con su jefe? } \\
\hline En otro edificio & & & & & & & & & & Al lado \\
\hline $0 \%$ & $10 \%$ & $20 \%$ & $30 \%$ & $40 \%$ & $50 \%$ & $60 \%$ & $70 \%$ & $80 \%$ & $90 \%$ & $100 \%$ \\
\hline
\end{tabular}

